\title{
The nuclear path of the CNO cycles in massive stars
}

\author{
N. Przybilla ${ }^{1}$, M. F. Nieva ${ }^{1}$, A. Maeder ${ }^{2}$ and G. Meynet ${ }^{2}$ \\ ${ }^{1}$ Institut für Astro- und Teilchenphysik, Universität Innsbruck, \\ Technikerstr. 25/8, A-6020 Innsbruck, Austria \\ email: norbert.przybilla@uibk.ac.at, maria-fernanda.nieva@uibk.ac .at \\ ${ }^{2}$ Geneva Observatory, University of Geneva, Maillettes 51, 1290 Sauverny, Switzerland \\ email: georges.meynet@unige.ch, andre.maeder@unige.ch
}

\begin{abstract}
We discuss how the nuclear path of the CNO cycles in massive stars can be employed as a quality indicator for model atmosphere analyses and for the derivation of tight observational constraints for developing a better understanding of the evolution of rotating massive stars.
\end{abstract}

Keywords. stars: abundances, stars: atmospheres, stars: early-type, stars: evolution, stars: fundamental parameters, supergiants

While a general understanding of the evolution and fate of massive stars has been developed over the past decades, many observational findings still lack a comprehensive explanation. This is because models of massive star evolution have to rely on many physical ingredients, and not all of them are known with confidence. Some of them can be constrained directly via observations in principle, e.g. mass-loss rates throughout the Hertzsprung-Russell diagram, while others are less accessible like diffusion coefficients or overshooting. At present, the latter have to be constrained empirically, by comparison of model predictions to observable effects they produce, traceable by asteroseismology or quantitative spectroscopy using model atmospheres. That is, the quality of stellar evolution models depends to some part on the quality of observational results put forward.

Quantitative spectroscopy, like any other technique of analysis or measurement, is inherently prone to be affected by systematic bias, which is typically hard to be identified. In order to reach high accuracy and precision in observational results, like on atmospheric and fundamental parameters of stars, or on elemental abundances, it would be highly useful to find ways to test for the presence of systematic bias.

Indeed, a simple and objective test to assess the accuracy and precision of analysis results exists, a consequence of the actions of the CNO cycles in massive stars, which sequentially transform one particle species into another, keeping the total of the CNO particles conserved (Przybilla et al. 2010; Maeder et al. 2014). The ratios of the participants in the CNO cycles at the stellar surface depend both on the changes produced by nuclear reactions and the dilution effects produced by mixing. Analytical relations can be derived for the predicted nuclear paths in the cases that the $\mathrm{CN}$ or $\mathrm{ON}$ cycles dominate. E.g., an initially almost linear slope of $\mathrm{d}(N / C) / \mathrm{d}(N / O) \approx 4$ is indicated for stars in the mass range up to $\sim 20-30 M_{\odot}$ (dominance of the $\mathrm{CN}$ cycle) in the $N / C$ vs. $N / O$ diagram, adopting pristine $\mathrm{CNO}$ abundances as found in the solar neighbourhood at present day (e.g. Nieva \& Przybilla 2012). This is confirmed by detailed stellar evolution models. In particular, the $N / C$ vs. $N / O$ diagram shows little dependence on the initial stellar masses, rotation velocities, and nature of the mixing processes (e.g. rotational, mass overflow in binaries) up to a relative enrichment of $N / O$ by a factor of about four. This means, the nuclear path of the $\mathrm{CNO}$ cycles is universal and essentially independent of 


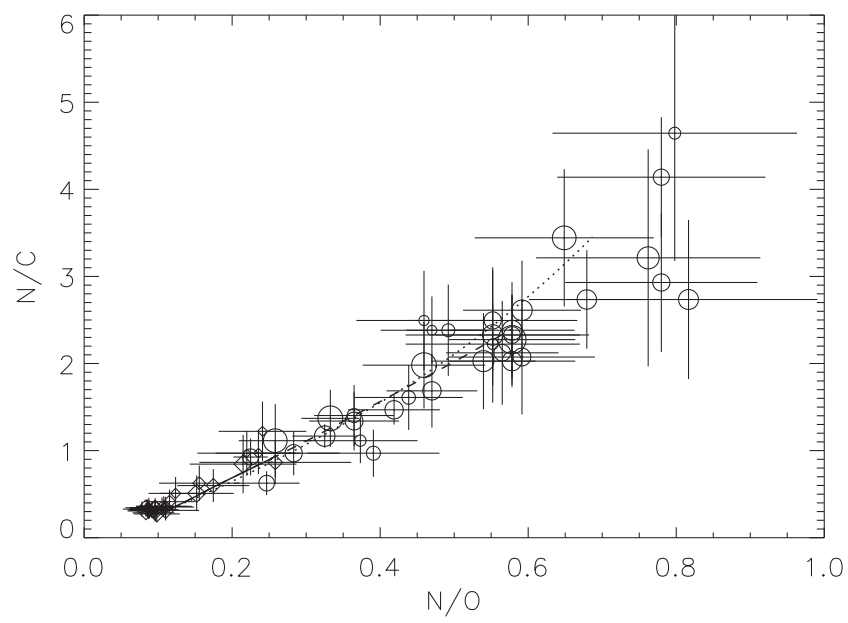

Figure 1. $N / C$ vs. $N / O$ abundance ratios (by mass) for homogeneously analysed stars from the samples of Przybilla et al. (2010), Nieva \& Przybilla (2012) and Nieva \& Simón-Díaz (2011), and including preliminary data on supergiants from the sample of Firnstein Przybilla (2012). B-type main-sequence stars are displayed as diamonds, BA-type supergiants as circles. The symbol size encodes the stellar mass (ranging from $\sim 6$ to $\sim 25 M_{\odot}$ ) and error bars give $1 \sigma$-uncertainties. The lines represent predictions from evolution calculations, for a rotating $15 M_{\odot}$ star, $v_{\text {rot }}^{\text {ini }}=300 \mathrm{~km} \mathrm{~s}^{-1}$ (Meynet \& Maeder 2003, solid line: until the end of the main sequence; dashed line: until the end of He burning) and for a star of the same mass and $v_{\text {rot }}^{\text {ini }}$ that in addition takes the interaction of rotation and a magnetic dynamo into account (Maeder \& Meynet 2005, dotted line: until the end of the main sequence). Note the clump of 20 stars at the base of the tracks that results from objects with pristine CNO abundances.

the model details, providing a robust, simple and objective test for observational results (provided that CNO abundances can be determined in the course of the analysis).

Figure 1 shows an example how tight the match between a homogeneously analysed star sample based on high-quality spectra and prediction by stellar evolution models can be. This is because observational bias and systematic error inherent to the analysis technique were minimised. Note that the larger error bars for the more mixed objects are a consequence of the linear scale chosen for the $N / O-N / C$ diagram, while the analysis gives about constant relative uncertainties. Note further, that the choice of the particular stellar evolution models was based on the good match of their initial ratios between CNO abundances with the observed ones. The nuclear paths predicted by more recent stellar evolution models like those of Ekström et al. (2012) show a very similar behaviour, but they are shifted because of different initial CNO ratios.

Acknowledgements. We would like to thank Patrick Eggenberger, Sylvia Ekström, Markus Firnstein and Cyril Georgy for fruitful discussions and contributions to this topic.

\section{References}

Ekström, S., Georgy, C., Eggenberger, P., et al. 2012, A\&A, 537, A146

Firnstein, M. \& Przybilla, N. 2012, A\& $A$, 543, A80

Maeder, A. \& Meynet, G. 2005, A\&A, 440, 1041

Maeder, A., Przybilla, N., Nieva, M. F., et al. 2014, A\& A, 565, A39

Meynet, G. \& Maeder, A. 2003, A\&A, 404, 975

Nieva, M. F. \& Przybilla, N. 2012, A\& $A$, 539, A143

Nieva, M. F. \& Simón-Díaz, S. 2011, A\& A, 532, A2 (NSD11)

Przybilla, N., Firnstein, M., Nieva, M. F., Meynet, G., \& Maeder, A. 2010, A\&6A, 517, A38 